\title{
Changes in cardiac cells due to ticagrelor and enoxaparin in a rat ischemia/reperfusion model
}

\author{
Orhan Fındık (1), Ozgur Baris²* (1), Yusufhan Yazir ${ }^{3,4}$ (1), Melda Yardimoglu Yilmaz ${ }^{4}$ (1), \\ Selenay Furat Rencber ${ }^{4} \mathbb{B}$, Kübra Kavram Sarihan ${ }^{4} \mathbb{C}$, Atike Tekeli Kunt ${ }^{5}$ (i)
}

\section{SUMMARY}

OBJECTIVE: Studies on ischemia/reperfusion injury remain the focus of interest. Ticagrelor and enoxaparin, which are antiaggregant and anticoagulant drugs developed for use in many cardiovascular pathologies, are still included in many ischemia/reperfusion studies. Remarkably, their new protective effects, especially with regard to ticagrelor, continue to be reported in the current literature. The aim of this study was to evaluate the beneficial effects of ticagrelor and enoxaparin pretreatments on the rat heart with histological and immunohistochemical markers in an ischemia/reperfusion model.

METHODS: Wistar-albino rats (weighing 350-400 g) were divided into four groups as follows: Sham-Control (Group 1), ControlSaline+ischemia/reperfusion (Group 2), Ticagrelor+ischemia/reperfusion (Group 3), and Enoxaparin+ischemia/reperfusion (Group 4). The ischemia/reperfusion injury model was applied to Group 2, Group 3 and Group 4. Heart tissue sections were stained with hematoxylin and eosin for histological examinations. Caspase 3 immunostaining was evaluated to detect apoptosis in the heart tissue sections.

RESULTS: Both pretreatments ameliorated the ischemic damage but especially tissue sections belonging to Group 3 were nearly similar to control levels. The results indicated that ischemia/reperfusion-induced myocardial damage was significantly increased in Group 2 , whereas ticagrelor and enoxaparin pretreatments in Group 3 and Group 4 significantly decreased apoptotic scores and the histological appearance of the Group 3 close to the normal myocardium ( $p<0.001)$.

CONCLUSION: As supported by histological findings in our study, ticagrelor and enoxaparin have protective properties for heart tissue in this ischemia/reperfusion injury model.

KEYWORDS: Ticagrelor. Enoxaparin. Ischemia. Rats. Reperfusion injury.

\section{INTRODUCTION}

"Ischemia" is defined as the loss of blood flow by depriving a tissue of metabolic substrates such as glucose as a result of reduced blood flow and impaired nutrition. "Reperfusion" is used to describe the restoration of blood flow and tissue nutrition after a period of ischemia and is a critical step in dealing with any ischemia condition ${ }^{1,2}$. Ischemia is associated with progressive decline in cellular functions, increased oxidative and inflammatory response. Increased permeability, disruption of extracellular matrix, increased proteases, impaired intravascular laminar flow, and the relationship among damaged endothelial-thrombus aggregation, leukocyte adhesion, foam cells, and ischemia/reperfusion (I/R) injury have been clarified at present ${ }^{1}$. In addition to their current primary effects in

\footnotetext{
${ }^{1}$ Health Sciences University, Derince Training and Research Hospital, Department of Cardiovascular Surgery - Kocaeli, Turkey.

${ }^{2}$ Kocaeli University, Faculty of Medicine, Department of Cardiovascular Surgery - Kocaeli, Turkey.

${ }^{3}$ Kocaeli University, Center for Stem Cell and Gene Therapies Research and Practice - Kocaeli, Turkey.

${ }^{4}$ Kocaeli University, Faculty of Medicine, Department of Histology and Embryology - Kocaeli, Turkey.

${ }^{5}$ Kirikkale University School of Medicine, Department of Cardiovascular Surgery - Kirikkale, Turkey.

*Corresponding author: drozgurbaris@gmail.com

Conflicts of interest: the authors declare there are no conflicts of interest. Funding: none.

Received on September 14, 2021. Accepted on September 16, 2021.
} 
cardiovascular therapy and protection, many pharmacological agents provide clues as to where the protective effects will be located in experimental animal studies. Therefore, studies on $\mathrm{I} / \mathrm{R}$ injury remain the focus of interest.

Ticagrelor and enoxaparin, which are antiaggregant and anticoagulant drugs developed for use in the treatment of many cardiovascular pathologies, occlusive and ischemic cardiovascular diseases, myocardial infarction (MI), some arrhythmias, ischemic stroke, valve surgery, and coronary bypass surgery, are still included in many I/R studies. Remarkably, the new protective effects of ticagrelor (it significantly prevents depolarization of mitochondrial membrane potential and increases in reactive oxygen species (ROS) with a marked increase in the ATP level in insulin-resistant $\mathrm{H}$ 9c2 cells and reverses the increases in the resting level of free $\mathrm{Ca} 2+$ and mRNA level of P2Y12 receptors as well as preserved endoplasmic reticulum stress and apoptosis) and enoxaparin (it reduces cell proliferation and tumor cell migration by inhibition of protease-activated receptor-1 [PAR-1]) continue to be reported in the current literature $^{3-5}$.

With the increase in the number and quality of qualified experimental basic education and research centers in recent years, immunohistochemical laboratory studies shed light on clinical studies. In this way, the protective effects of pharmacological agents on cells and systems became more important. It should be emphasized that the effects of many pharmacological agents on inflammation, apoptosis and I/R injury have been better studied with multidisciplinary precursor animal experiments conducted in these qualified centers.

The aim of this study was to evaluate the beneficial effects of ticagrelor and enoxaparin pretreatments on the rat heart with histological and immunohistochemical markers in an I/R model.

\section{METHODS}

\section{Animal preparation and experimental design}

All experiments were carried out in accordance with the European Communities Council Directive of November 24, 1986 (86/609/ EEC). The experiments reported in this study were conducted in accordance with the Regulation of Animal Research Ethics Committee in Turkey (July 6, 2006, Number 26220). The ethical approval was granted by the Kocaeli University Animal Experiments Ethics Committee. All experiments were conducted between 9:00 a.m. and 12:00 p.m. under standard laboratory conditions $\left(22 \pm 2^{\circ} \mathrm{C}\right.$ room temperature; $12 \mathrm{~h} \mathrm{light/dark} \mathrm{cycle}$ with lights on at 7:00 a.m.). Tap water and food pellets were provided ad libitum for animals.
Thirty-six mature male Wistar-albino rats (weighing 350$400 \mathrm{~g}$ ) were divided into four groups as follows: Sham-Control (Group1), Control-Saline+I/R (Group 2), Ticagrelor+I/R (Group 3), and Enoxaparin+I/R (Group 4). The I/R injury model was applied to Group 2, Group 3, and Group 4 induced by clamping the aorta infrarenally for $2 \mathrm{~h}$ followed by $4 \mathrm{~h}$ of reperfusion after anesthetized with intraperitoneal ketamine hydrochloride (Ketalar, Pfizer, Istanbul, Turkey). Cessation of arterial flow was confirmed by means of the absence of an audible continuous-wave Doppler signal. Group 1 was not treated. Before the ischemic period, Group 2 received $0.1 \mathrm{~mL} / \mathrm{kg}$ saline, Group 3 received $25 \mathrm{mg} / \mathrm{kg}$ ticagrelor (Brilinta, AstraZeneca, Södertalje, Sweden) via gastric gavage while Group 4 received $0.75 \mathrm{mg} / \mathrm{kg}$ enoxaparin (Clexane, Sanofi Winthrop Industrie, Maisons-Alfort, France) subcutaneously ${ }^{6,7}$.

At the end of the experiment, rats were sacrificed by the lethal dose of sodium thiopental (Pentothal Sodium, Abbot, Italy). The hearts of all rats were excised through midline sternotomy, washed with $0.9 \%$ saline solution, and then fixed in $10 \%$ neutral buffered formalin for histological tissue processing.

\section{Hematoxylin and eosin staining}

Heart tissue samples were dehydrated in graded series of ethanol, cleared in xylene, and embedded in paraffin blocks. Notably, $4 \mu \mathrm{m}$-thick sections were stained with $\mathrm{H} \& \mathrm{E}$ for histological examinations. Degeneration of cardiomyocyte arrangement, heterogeneity of sarcoplasm, interfibrillar distance, necrosis, and increase in capillaries were assessed.

\section{Caspase-3 immunohistochemistry}

Caspase-3 immunostaining was evaluated to detect apoptosis in the heart tissue sections. In brief, tissue sections were deparaffinized with xylene and then rehydrated. Following the antigen retrieval step, the sections were incubated in $3 \% \mathrm{H}_{2} \mathrm{O}_{2}$ for 15 min to block endogenous peroxidase activity.

After blocking serum, the anti-caspase- 3 monoclonal antibody (74T2, Life Technologies/Thermo, 1:100 dilution) was added to the sections and incubated overnight at room temperature. After washing with phosphate-buffered saline (PBS), the biotinylated secondary antibody (ab80437, Abcam) was added to the sections and incubated for $20 \mathrm{~min}$. Then, the sections were washed with PBS and incubated with horseradish peroxidase (HRP)-labeled streptavidin at room temperature for 15 min. The samples were developed using 3,3'-diaminobenzidine (DAB) chromogen according to the manufacturer's standard procedure. Negative control staining was applied by omitting the primary antibody. The sections were counterstained using hematoxylin for $1 \mathrm{~min}$ and examined at 200x and 400x magnification using a light microscope. Positive cells displayed 
a brown-yellow coloration. The approximate percentage of apoptotic cells was estimated based on 10 representative fields in each section. The immunostaining intensity was scored as negative (-), very weak (1+), moderate (2), strong (3+), and very strong $(4+)^{7-10}$.

The total score was based on the percentage of positive cells and the degree of immunostaining intensity. The percentage of positive cells was graded as follows: no stained cells (Grade 0), 1-25\% stained cells (Grade 1), 26-50\% stained cells (Grade 2), 51-75\% stained cells (Grade 3), and $76-100 \%$ stained cells (Grade 4) in the representative field. Three observers who were blinded to the groups performed evaluations semi-quantitatively. The total index was calculated using the following formula: total index (percentage of positive cells) $\times$ (immunostaining intensity). All slides were examined under a light microscope (Leica DM 1000, Germany), and photomicrographs were taken using an attached camera (Leica DMC 2900, Germany).

\section{Statistical analysis}

All statistical analyses were performed using IBM SPSS for Windows version 20.0 (IBM Corp., Armonk, NY, USA). Caspase-3 scoring was analyzed using this software and presented as meantstandard deviation (SD). The KolmogorovSmirnov test was used for evaluating the normal distribution of the variables. The difference between the groups was analyzed by using a one-way analysis of variance (ANOVA) for numerical variables with normal distribution. Tests were performed within a $95 \%$ confidence interval and $\mathrm{p}<0.05$ was considered significant.

\section{RESULTS}

\section{Histological results}

H\&E staining of the myocardial sections of the Group 1 showed normal morphology (Figure 1A), whereas Group 2 exhibited disorganized cardiac muscle fibers, heterogeneity of sarcoplasm, morphologically deformation of nuclei, increase in interfibrillar space, edematous areas in the connective tissue, necrosis, and increased capillary formation (Figure 1B). However, both pretreatments improved and ameliorated the ischemic damage but especially tissue sections belonging to Group 3 were nearly similar to control levels (Figures 1C and 1D).

\section{Caspase-3 immunoreactivity}

In addition to morphological findings, caspase- 3 immunoreactivity in myocardium sections belonging to the Group 1, Group 2, and pretreatment groups was examined (Figure 2).

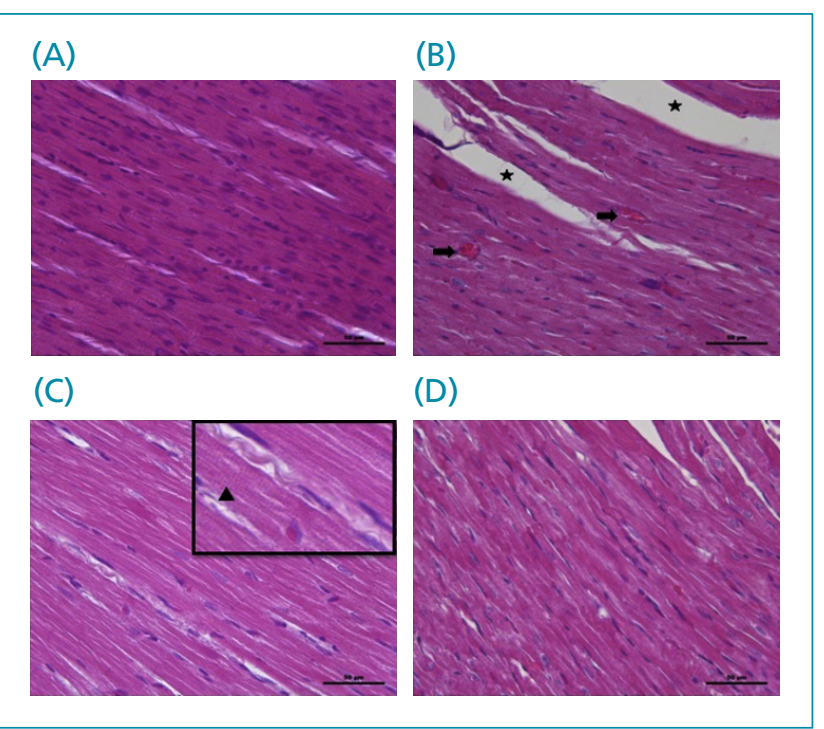

Figure 1. Hematoxylin and eosin staining of the myocardial sections of the Sham-Control (A), Control-Saline+ischemia/ reperfusion (B), Ticagrelor+ischemia/reperfusion (C), and Enoxaparin+ischemia/reperfusion (D). Note degeneration of cardiomyocyte arrangement and heterogeneity of sarcoplasm, interfibrillar distance (star), increased capillaries (arrow) in Control-Saline+ischemia/reperfusion group. Arrowhead indicates normal striated appearance. Hematoxylin and eosin $400 x$.

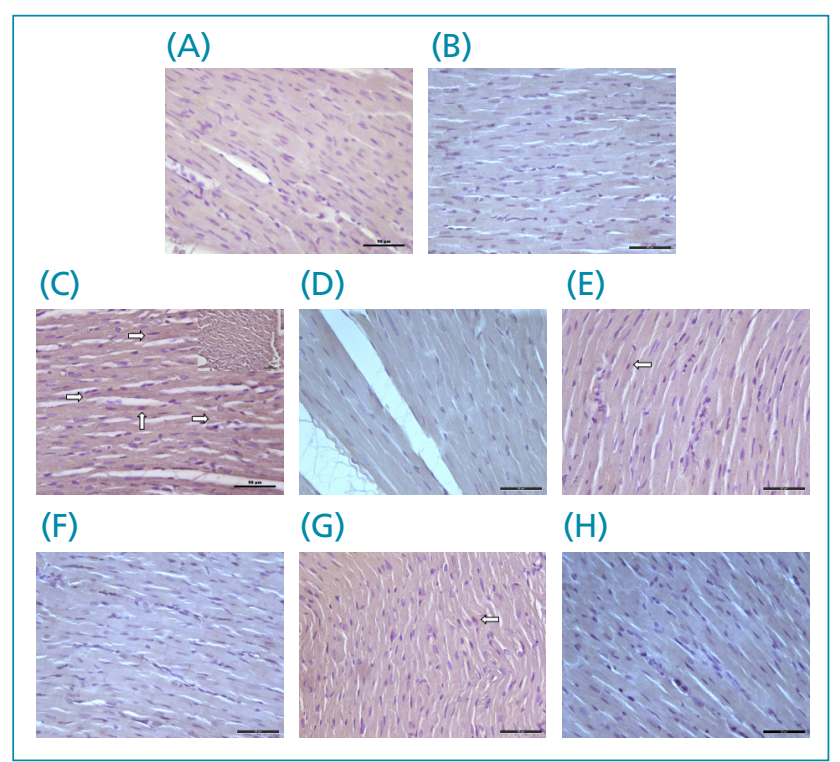

Figure 2. Caspase 3 immunoreactivity of the ShamControl (A), Control-Saline+ischemia/reperfusion (C), Ticagrelor+ischemia/reperfusion (E), and Enoxaparin+ischemia/ reperfusion (G) groups with their negative controls (B, D, $\mathrm{F}, \mathrm{H})$. In (C), note the longitudinal sections of dark-stained cardiomyocytes and their transverse sections in the upper right box. Arrows indicate the Caspase 3 (+) cardiomyocytes. In right side, no immunoreactivity is observed in negative controls. $400 x$. 
Increased caspase- 3 immunoreactivity was noticed in the myocardium sections of Group 2, and caspase-3 immunostaining was weaker in the pretreatment groups (Figure 2).

Caspase-3 expression was significantly higher in Group 2 compared with Group $1(\mathrm{p}<0.001)$, and this expression index was significantly decreased in the pretreatment groups compared with Group 2 ( $\mathrm{p}<0.001$; Figure 3). For all groups, caspase-3 negative control slides were examined, and no immunoreactivity was observed $(\mathrm{p}<0.001$; Figure 3$)$.

Caspase-3 total IHC index was significantly decreased in ticagrelor and enoxaparin pretreatment groups compared with the Control-Saline+I/R group ( $\mathrm{p}<0.001)$. These results indicated that $\mathrm{I} / \mathrm{R}$-induced myocardial damage was significantly increased in Group 2, whereas ticagrelor and enoxaparin pretreatments in Group 3 and Group 4 significantly decreased caspase-3 scores (Figure 3).

In conclusion, the apoptotic index with the histological appearance of the Group 3 and Group 4 close to the normal myocardium display that ticagrelor and enoxaparin have effective protective properties for the heart muscle tissue in this $I / R$ injury model.

\section{DISCUSSION}

This study showed that ticagrelor and enoxaparin pretreatments have cardioprotective effects on myocardium in the $I / R$ rat model. According to our findings and previous data, ticagrelor possibly increased the extracellular adenosine which is a major mediator of myocardial protection against I/R injury and is essential for myocardial protection by ischemic preconditioning and various pharmacological preconditioning ${ }^{11}$.

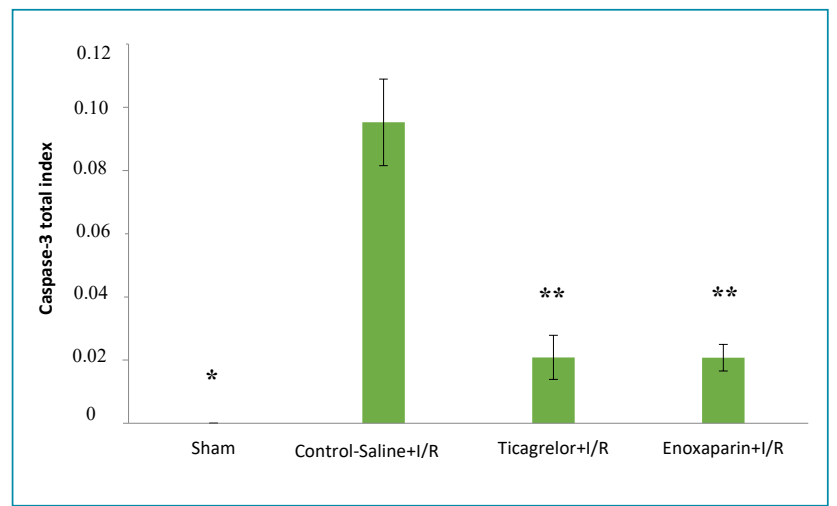

Figure 3. Graphical representation of Caspase-3 total index. While caspase-3 total index was significantly higher in Group 2 compared with Group 1 (* $p<0.001)$, this index was significantly decreased in the pretreatment groups (Group 3 and Group 4) compared with Group $2\left({ }^{* *} p<0.001\right)$.
Abdominal aortic cross-clamping is often used for I/R injury in experimental animal models. I/R injury affects not only the kidney, testes, and lower extremity distal to the clamped abdominal aorta but also proximal and distant tissues such as the brain, heart, and lungs, with a series of inflammatory cascades triggered ${ }^{12}$. The mechanisms that cause I/R injury after ischemia are as follows:

(i) increased amount of $\mathrm{Ca}^{2+}$ ions entering the myocardial cells in the ischemic process;

(ii) the excessive amount of hypoxanthine accumulated in the issue as a result of adenosine triphosphate (ATP) breakdown during the ischemic period, to form excess superoxide by xanthine oxidase and the formation of free oxygen radicals; and

(iii)increased thromboxane-A2 level.

Reperfusion is responsible for major lesions seen in the cells of the ischemic organ. Systemic complications, such as adult respiratory distress syndrome and kidney and liver dysfunction, can be seen with this cellular damage. Occasionally, these negative effects are seen through the systemic blood circulation, by leukocytes, proinflammatory cytokines, adhesions, and reactive oxygen metabolites, affecting more than one distant organ and systems ${ }^{13-16}$. We know that the endothelium helps to prevent cell-cell interactions between blood-borne inflammatory cells (i.e., leukocytes and platelets). Adenosine and nitroxide $\left(\mathrm{NO}^{-}\right)$, secreted from the endothelium and showing antineutrophil and antiplatelet effects, cannot be secreted sufficiently from the endothelium as a result of $\mathrm{I} / \mathrm{R}$ damage. Inflammatory reactions with free oxygen radicals and proteases are further increased with resisting neutrophilic activation and proinflammatory cytokines such as interleukin-1 (IL-1), IL-6, and complement activity such as $\mathrm{C} 3 \mathrm{a}, \mathrm{C} 5 \mathrm{a}$ begin the process leading to cell death ${ }^{17}$.

The cardiac dysfunction occurs in myocardial ischemia, and the loss of energy substrates in myocardial ischemia leads to the generation of ROS; the high levels of ROS are deleterious and can induce a variety of cardiomyocyte abnormalities ${ }^{18}$. Oxidative stress and the generation of ROS during I/R increase the production of proinflammatory cytokines such as tumor necrosis factor-alpha (TNF- $\alpha$ ), IL-6, and IL-1 $\beta$. These cytokines induce apoptosis and cellular damage. ROS are potent oxidizing and reducing agents, which activate neutrophil and lipid peroxidation, leading to cell membrane damage ${ }^{19}$. In the study by Shiroma ME et al. ${ }^{20}$, it has been shown that melatonin can support better quality ovarian grafts in inflammatory processes and oxidative stress and explained that the purpose of cell protection is to prevent mitochondrial apoptosis by reducing bcl 2 expression in I/R injuries that damage cells and mitochondria ${ }^{20}$. 
Ticagrelor, a cyclopentyl triazolo pyrimidine, is a direct-acting, selective, and reversibly binding $\mathrm{P} 2 \mathrm{Y}_{12}$ receptor antagonist. The $\mathrm{P} 2 \mathrm{Y}_{12}$ receptor has a major role in primary hemostasis and in arterial thrombosis. When bound to the $\mathrm{P}_{2} \mathrm{Y}_{12}$ receptor, ticagrelor prevents adenosine diphosphate (ADP)mediated platelet activation and aggregation. In addition to the primary effects mediated by $\mathrm{P}_{2} \mathrm{Y}_{12}$ receptor antagonism, ticagrelor increases local endogenous adenosine levels by inhibiting the uptake of adenosine $\mathrm{e}^{21}$, thereby protecting the extracellular adenosine from its intracellular metabolism. Unlike thienopyridines, ticagrelor is not a prodrug. The antiplatelet effect is independent of the CYP2C19 pathway. In many $\mathrm{I} / \mathrm{R}$ studies, adenosine and $\mathrm{NO}^{-}$were used against this cascade in preventing the increased inflammatory response seen during cardiopulmonary bypass, and their positive effects were demonstrated ${ }^{22}$. Thus, by increasing its production and preventing its metabolism, ticagrelor should have beneficial effects on extracellular adenosine levels ${ }^{23}$. Besides, several clinical studies have supported the safety, tolerability, and efficacy of ticagrelor in the setting of stable coronary disease and acute MI.

The platelet inhibition and patient outcomes (PLATO) trial showed that ticagrelor was associated with a lower incidence of cardiovascular mortality, MI, or stroke compared with the alternative $\mathrm{P}_{2} \mathrm{Y}_{12}$ antagonist clopidogrel in patients with acute coronary syndromes when given on a background therapy of acetylsalicylic acid ${ }^{24,25}$. Although the difference was originally ascribed to better and more consistent platelet inhibition than with clopidogrel, it has been speculated that additional benefit of ticagrelor might be provided via an adenosine mediated mechanism ${ }^{26}$. In addition, a post hoc analysis of the PLATO trial has suggested that ticagrelor reduces sudden death and has also been shown to lower the risk of recurrent ischemic events, including cardiovascular and coronary heart disease death, in diabetic patients with a history of $\mathrm{MI}^{11}$.

Enoxaparin is a heparin with low molecular weight, and it is an antithrombotic drug that is used in the treatment of thromboembolism, ischemia, and infarction. Enoxaparin inhibits the conversion of prothrombin to thrombin and reduces the conversion of fibrinogen to fibrin, preventing clot formation. It also reduces coagulation factors and inactivates factor- $\mathrm{X}$, and enoxaparin has been shown to exert powerful antioxidant effects by preventing lipid peroxidation. Furthermore, this drug demonstrated anti-inflammatory benefits. In the study by Shaker et al., enoxaparin treatment was shown to effectively reduce doxorubicin-induced cardiotoxicity by suppressing oxidative stress and inflammation and preventing apoptosis ${ }^{27}$. Shaker et al. showed that in the doxorubicin group, there was an increase in malondialdehyde (MDA), a marker of oxidative stress, and a decrease in total antioxidant capacity, increased levels of inflammatory markers such as TNF-a and IL-1 $\beta$ and an apoptotic marker, caspase- $3^{27}$. Additionally, in this study, although enoxaparin treatment did not completely reverse doxorubicin-induced cardiotoxic damage, it decreased cardiac troponin-I compared with the doxorubicin group and improved cardiomyopathy histopathological scores; it reduced MDA levels, increased the total antioxidant capacity in the rat heart to comparable levels compared with the control group; and it significantly decreased TNF- $\alpha$, IL- $1 \beta$, and caspase- 3 levels compared with only doxorubicin group ${ }^{27}$. However, there are not enough studies in the literature regarding the effect of enoxaparin on myocardial I/R damage. In our study, only the apoptotic activity/caspase-3 score and histological evaluation and focus on apoptosis at the histological level can be considered as limiting factors.

In contrast, the protective effect of ticagrelor, which we showed in our study, overlaps with the study by Liu X et al., and the cardioprotective effects of ticagrelor might partly be mediated by downregulating galectin-3 expression in infarct area in a rat model of myocardial I/R injury ${ }^{3}$.

Myocyte regeneration and death of myocytes occur physiologically. Recent studies of these cellular processes increase in pathological conditions have challenged the idea that the heart is a postmitotic organ. The ideas that cardiac homeostasis can be regulated by multipotent cardiac stem cells are guiding new studies ${ }^{28}$. Due to that, comprehensive research on cardiac stem cells will only be possible with a thorough understanding of the inflammatory response and the $\mathrm{I} / \mathrm{R}$ cascade.

According to the study by Birnbaum et al. ${ }^{29}$, ticagrelor, possibly by inducing a local increase in extracellular adenosine levels, may augment reparative mechanisms that attenuate adverse remodeling and fibrosis, and it may increase cell proliferation and stem cell recruitment. Based on these data, it may provide an alternative approach to cardioprotection and stem cell therapy, as evidenced by improved cardiac function ${ }^{29}$.

\section{CONCLUSIONS}

Ticagrelor and enoxaparin pretreatments have cardioprotective effects on the myocardium in the I/R rat model. According to our findings and previous data, ticagrelor possibly increased the extracellular adenosine which is a major mediator of myocardial protection against ischemia-reperfusion injury and is essential for the myocardial protection by ischemic preconditioning and various pharmacological preconditioning. ${ }^{5}$. However, 
more comprehensive experimental and clinical studies should be performed to figure out the underlying mechanisms. In conclusion, these results we showed in our study may guide to the uncovered aspects of the $\mathrm{I} / \mathrm{R}$ cascade, advanced experimental and cardiac stem cell studies to be performed with anticoagulant drugs and antiaggregant drugs. With this laborious study on the I/R model in rat heart, we hope that the protective effect of ticagrelor and enoxaparin may make an effective contribution to larger-scale studies, particularly cardiac and vascular pathologies.

\section{AUTHORS' CONTRIBUTIONS}

OF: Conceptualization, Project administration, Data curation, Investigation, Methodology, Writing - original draft. OB: Conceptualization, Project administration, Data curation, Formal Analysis, Writing - original draft, Writing - review \& editing. YY: Investigation, Supervision, Validation. MYY: Methodology, Resources, Writing - review \& editing. SFR: Visualization, Writing - original draft. KKS: Formal Analysis, Software. ATK: Conceptualization, Project administration, Methodology, Supervision.

\section{REFERENCES}

1. Fitridge $R$, Thompson $M$, editors. Mechanisms of vascular disease: a reference book for vascular specialists [Internet]. Adelaide: University of Adelaide Press; 2011. PMID: 30484990

2. Brandão RI, Gomes RZ, Lopes L, Linhares FS, Vellosa JCR, Paludo KS. Remote post-conditioning and allopurinol reduce ischemia-reperfusion injury in an infra-renal ischemia model. Ther Adv Cardiovasc Dis. 2018;12(12):341-9. https://doi. org/10.1177/1753944718803309

3. Liu X, Gu Y, Liu Y, Zhang M, Wang Y, Hu L. Ticagrelor attenuates myocardial ischaemia-reperfusion injury possibly through downregulating galectin-3 expression in the infarct area of rats. Br J Clin Pharmacol. 2018;84(6):1180-6. https:// doi.org/10.1111/bcp.13536

4. Olgar Y, Tuncay E, Billur D, Durak A, Ozdemir S, Turan B. Ticagrelor reverses the mitochondrial dysfunction through preventing accumulated autophagosomes-dependent apoptosis and ER stress in insulin-resistant H9c2 myocytes. Mol Cell Biochem. 2020;469(1-2):97-107. https://doi.org/10.1007/ s11010-020-03731-9

5. Alturkistani A, Ghonem N, Power-Charnitsky VA, Pino-Figueroa A, Migliore MM. Inhibition of PAR-1 receptor signaling by enoxaparin reduces cell proliferation and migration in A549 cells. Anticancer Res. 2019;39(10):5297-310. https://doi. org/10.21873/anticanres.13723

6. Findik O, Kunt AT, Yazir Y, Yardimoğlu M, Yilmaz SG, Aydin U, et al. Ticagrelor attenuates apoptosis of lung and myocardial cells induced by abdominal aorta ischemia/reperfusion. In Vivo. 2016;30(3):243-9. PMID: 27107082

7. Fındık O, Yılmaz MY, Yazır Y, Rençber SF, Sarıhan KK, Kunt AT. Investigation of the protective effect of enoxaparin and ticagrelor pretreatment against ischemia-reperfusion injury in rat lung tissue. Rev Assoc Med Bras (1992). 2019;65(9):1193200. https://doi.org/10.1590/1806-9282.65.9.1193

8. van Diest PJ, van Dam P, Henzen-Logmans SC, Berns E, van der Burg ME, Green J, et al. A scoring system for immunohistochemical staining: consensus report of the task force for basic research of the EORTC-GCCG. European Organization for Research and Treatment of CancerGynaecological Cancer Cooperative Group. J Clin Pathol. 1997;50(10):801-4. https://doi.org/10.1136/jcp.50.10.801

9. Panzan MQ, Mattar R, Maganhin CC, Simões RS, Rossi AG, Motta EL, et al. Evaluation of FAS and caspase-3 in the endometrial tissue of patients with idiopathic infertility and recurrent pregnancy loss. Eur J Obstet Gynecol
Reprod Biol. 2013;167(1):47-52. https://doi.org/10.1016/j. ejogrb.2012.10.021

10. Fuchs LFP, Veras MM, Saldiva PHN, Sasso GRDS, Carvalho $K C$, Simões MJ, et al. Ambient levels of concentrated PM2.5 affects cell kinetics in adrenal glands: an experimental study in mice. Gynecol Endocrinol. 2017;33(6):490-5. https://doi. org/10.1080/09513590.2017.1291617

11. Vilahur G, Gutiérrez M, Casani L, Varela L, Capdevila A, PonsLladó $\mathrm{G}$, et al. Protective effects of ticagrelor on myocardial injury after infarction. Circulation. 2016;134(22):1708-19. https://doi.org/10.1161/CIRCULATIONAHA.116.024014

12. Gozdzik W, Zielinski S, Zielinska M, Ratajczak K, Skrzypczak $P$, Rodziewicz $S$, et al. Beneficial effects of inhaled nitric oxide with intravenous steroid in an ischemia-reperfusion model involving aortic clamping. Int J Immunopathol Pharmacol. 2018;32:394632017751486. https://doi. org/10.1177/0394632017751486

13. Wu MY, Yiang GT, Liao WT, Tsai AP, Cheng YL, Cheng PW, et al. Current mechanistic concepts in ischemia and reperfusion injury. Cell Physiol Biochem. 2018;46(4):1650-67. https://doi. org/10.1159/000489241

14. Kalogeris T, Baines CP, Krenz M, Korthuis RJ. Cell biology of ischemia/reperfusion injury. Int Rev Cell Mol Biol. 2012;298:229317. https://doi.org/10.1016/B978-0-12-394309-5.00006-7

15. Loerakker S, Oomens CW, Manders E, Schakel T, Bader DL, Baaijens FP, et al. Ischemia-reperfusion injury in rat skeletal muscle assessed with T2-weighted and dynamic contrastenhanced MRI. Magn Reson Med. 2011;66(2):528-37. https:// doi.org/10.1002/mrm.22801

16. Ciz M, Cizova H, Lojek A, Kubala L, Papezikova I. Ischemia/ reperfusion injury of rat small intestine: the effect of allopurinol dosage. Transplant Proc. 2001;33(5):2871-3. https://doi. org/10.1016/s0041-1345(01)02223-0

17. Jordan JE, Zhao ZQ, Vinten-Johansen J. The role of neutrophils in myocardial ischemia-reperfusion injury. Cardiovasc Res. 1999;43(4):860-78. https://doi.org/10.1016/s00086363(99)00187-x

18. Levraut J, Iwase $H$, Shao ZH, Vanden Hoek TL, Schumacker PT. Cell death during ischemia: relationship to mitochondrial depolarization and ROS generation. Am J Physiol Heart Circ Physiol. 2003;284(2):H549-58. https://doi.org/10.1152/ ajpheart.00708.2002

19. Cure MC, Cure E, Kalkan Y, Tumkaya L, Aydin I, Kirbas A, et al. The protective effect of adalimumab on renal injury in a 
model of abdominal aorta cross-clamping. Adv Clin Exp Med. 2016;25(2):219-26. https://doi.org/10.17219/acem/33250

20. Shiroma ME, Damous LL, Cotrim FP, Roa CL, Cipolla-Neto J, Reiter RJ, et al. Pretreatment with melatonin improves ovarian tissue cryopreservation for transplantation. Reprod Biol Endocrinol. 2021;19(1):17. https://doi.org/10.1186/ s12958-021-00705-4

21. Al-Salama ZT, Keating GM, Keam SJ. Ticagrelor: a review in long term secondary prevention of cardiovascular events. Drugs. 2017;77(18):2025-36. https://doi.org/10.1007/s40265017-0844-8

22. Vinten-Johansen J, Zhao ZQ. Cardioprotection from ischemicreperfusion injury by adenosine. In: Abd-Elfattah AS, Wechsler AS, editors. Purines and myocardial protection. Boston/ Dordrecht/London: Kluwer Academic Publishers; 1995. p.31544.

23. Birnbaum Y, Birnbaum GD, Birnbaum I, Nylander S, Ye Y. Ticagrelor and rosuvastatin have additive cardioprotective effects via adenosine. Cardiovasc Drugs Ther. 2016;30(6):53950. https://doi.org/10.1007/s10557-016-6701-2

24. Wallentin L, Becker RC, Budaj A, Cannon CP, Emanuelsson $\mathrm{H}$, Held $\mathrm{C}$, et al. Ticagrelor versus clopidogrel in patients with acute coronary syndromes. N Engl J Med. 2009;361(11):104557. https://doi.org/10.1056/NEJMoa0904327
25. Cannon $C P$, Harrington RA, James $S$, Ardissino D, Becker $\mathrm{RC}$, Emanuelsson $\mathrm{H}$, et al. Comparison of ticagrelor with clopidogrel in patients with a planned invasive strategy for acute coronary syndromes (PLATO): a randomized doubleblind study. Lancet. 2010;375(9711):283-93. https://doi. org/10.1016/S0140-6736(09)62191-7

26. Cattaneo M, Schulz R, Nylander S. Adenosine-mediated effects of ticagrelor: evidence and potential clinical relevance. J Am Coll Cardiol. 2014;63(23):2503-9. https://doi.org/10.1016/j. jacc.2014.03.031

27. Shaker RA, Abboud SH, Assad HC, Hadi N. Enoxaparin attenuates doxorubicin induced cardiotoxicity in rats via interfering with oxidative stress, inflammation and apoptosis. BMC Pharmacol Toxicol. 2018;19(1):3. https://doi.org/10.1186/ s40360-017-0184-z

28. Anversa $P$, Leri $A$, Kajstura J. Cardiac regeneration. J Am Coll Cardiol. 2006;47(9):1769-76. https://doi.org/10.1016/j. jacc.2006.02.003

29. Birnbaum Y, Tran D, Chen H, Nylander S, Sampaio LC, Ye Y. Ticagrelor improves remodeling, reduces apoptosis, inflammation and fibrosis and increases the number of progenitor stem cells after myocardial infarction in a rat model of ischemia reperfusion. Cell Physiol Biochem. 2019;53(6):96181. https://doi.org/10.33594/000000189 